\title{
Image quality dependence on image processing software in computed radiography
}

\author{
Lourens J Strauss, BMedSc (Hons) (Medical Physics) \\ William I D Rae, MB BCh, PhD (Medical Physics)
}

Department of Medical Physics, University of the Free State, Bloemfontein

Corresponding author: L Strauss (lourens.strauss@gmail.com)

Background. Image post-processing gives computed radiography (CR) a considerable advantage over film-screen systems. After digitisation of information from CR plates, data are routinely processed using manufacturer-specific software. Agfa CR readers use MUSICA software, and an upgrade with significantly different image appearance was recently released: MUSICA ${ }^{2}$.

Aim. This study quantitatively compares the image quality of images acquired without post-processing (flatfield) with images processed using these two software packages.

Methods. Four aspects of image quality were evaluated. An aluminium step-wedge was imaged using constant $\mathrm{mA}$ at tube voltages varying from 40 to $117 \mathrm{kV}$. Signal-to-noise ratios (SNRs) and contrast-to-noise ratios (CNRs) were calculated from all steps. Contrast variation with object size was evaluated with visual assessment of images of a Perspex contrast-detail phantom, and an image quality figure (IQF) was calculated. Resolution was assessed using modulation transfer functions (MTFs).

Results. SNRs for MUSICA ${ }^{2}$ were generally higher than the other two methods. The CNRs were comparable between the two software versions, although MUSICA ${ }^{2}$ had slightly higher values at lower $\mathrm{kV}$. The flatfield CNR values were better than those for the processed images. All images showed a decrease in CNRs with tube voltage. The contrast-detail measurements showed that both MUSICA programmes improved the contrast of smaller objects. MUSICA ${ }^{2}$ was found to give the lowest (best) IQF; MTF measurements confirmed this, with values at $3.5 \mathrm{lp} / \mathrm{mm}$ of $10 \%$ for MUSICA 2 , 8\% for MUSICA and 5\% for flatfield.

Conclusion. Both MUSICA software packages produced images with better contrast resolution than unprocessed images. MUSICA $^{2}$ has slightly improved image quality than MUSICA.

S Afr J Rad 2012;16(2):44-48.

\section{Introduction}

In radiology, image quality is a measure of the effectiveness with which clinical diagnoses may be made. It is usually evaluated on the basis of three measures of imaging performance, namely: spatial resolution, contrast, and noise. ${ }^{1}$ Other factors considered are the effect of patient dose on the image quality, as well as the occurrence of artefacts. Since the introduction of digital technology to radiology, many additional benefits have been realised. Probably the most important of these has been the ability to post-process and manipulate images. To readily obtain the most information from an image, manufacturers have developed optimised software algorithms to prepare images for soft copy display and reporting. The software has specific settings and filters which are used to achieve optimal images in an attempt to make the most accurate diagnosis. These settings are applied as appropriate for the specific views and anatomy being imaged, and include edge enhancement, contrast enhancement, excess contrast reduction, noise reduction, etc. Making accurate and efficient diagnoses from radiological images requires good image contrast and sufficient spatial resolution at acceptable noise levels, which is what manufacturers are striving to achieve. ${ }^{2}$

The software package developed by Agfa and used in Agfa CR readers is called MUSICA; an upgrade was recently released: MUSICA ${ }^{23,4}$ The acronym stands for Multi Scale Image Contrast Amplification, and the algorithm is essentially a multi-scale transform of the image data into a stack of detail layers. This is done in MUSICA using a Laplacian pyramid decomposition. ${ }^{5}$ Contrast can then be improved in each sub-band of spatial frequencies. Another important function of the processing software is to extract the appropriate signal sub-range through a process of signal normalisation. The unprocessed data captured by the CR plates, which are typically in the range of 211 gray levels, are reduced to only 28 gray levels for the observer to see most of the relevant image features at the same time. A detailed discussion of the image processing involved is presented by Vuylsteke et al. ${ }^{5}$

Image quality evaluation studies of CR systems typically use metrics such as contrast-to-noise ratios (CNRs), limiting spatial resolution, the modulation transfer function (MTF), noise power spectrum (NPS) and detective quantum efficiency (DQE). However, when evaluating only a part of the imaging chain (e.g. assessing the processing software), these metrics are not all relevant. Aspects which can be readily evaluated are signal-to-noise ratios (SNRs), contrast-to-noise ratios (CNRs), object visibility with decreasing size and contrast, and spatial resolution response using MTFs.

The signal-to-noise ratio within a specified region of interest (ROI) is defined by the following equation: ${ }^{1}$

$$
S N R=\frac{N}{\sigma}
$$

where $N$ is the average pixel value and $\sigma$ the standard deviation (noise) over the pixel values within the ROI.

Likewise, for the specified ROI, the contrast-to-noise ratio is given by equation 2 :

$$
C N R=\frac{A-B}{\sigma}
$$


where $A$ and $B$ are the average pixel values within the ROIs on adjacent steps on the image of the aluminium step wedge.

Object visibility with decreasing size and contrast can be evaluated qualitatively and/or using an image quality figure (IQF) score (visually assessed) specific for the in-house manufactured phantom. This is given by equation $3:{ }^{6}$

$$
I Q F=\sum_{i=1}^{n}\left(C_{i} \times D_{i, \min }\right)
$$

where $D_{i, \text { min }}$ the threshold diameter in the $i^{\text {th }}$ contrast column for columns, and $C_{i}$ is the depth of the hole generating the contrast in that column. Therefore, a low IQF represents better image quality.

The modulation transfer function of a system gives a very thorough description of its resolving capabilities, showing the fraction of an object's contrast recorded by the system as a function of the object's size (spatial frequency). ${ }^{1}$ It is calculated mathematically from the Fourier transform of the line spread function (LSF), which is the receptor's response to a thin, high contrast line. The Fourier transform of this LSF shows the system's effectiveness in transferring the different frequency components in an object into the image of that object.

The aim of this study was to quantitatively compare the image quality of images acquired without post-processing (flatfield) with images processed with Agfa's MUSICA and MUSICA ${ }^{2}$ software packages.

\section{Materials and method}

Data processing was done on images in DICOM format, using Matlab. ${ }^{7}$ All imaging was done on a standard X-ray machine at a fixed SSD of 100 $\mathrm{cm}$. Owing to the number of images acquired using low $\mathrm{kV}$ settings, the $\mathrm{CR}$ plates were placed on the bed to prevent the appearance of the ion chambers on the images. No scatter grid was present during imaging. Each image was repeated on three separate CR-plates of $24 \mathrm{x} 30 \mathrm{~cm}$ and read out on an Agfa CR 85-X digitiser using the three different postprocessing software algorithms.

An aluminium step-wedge placed on top of a $2.5 \mathrm{~cm}$ Perspex sheet was imaged at tube voltages of 40,55, 70, 85, 102 and $117 \mathrm{kV}$. The $\mathrm{mA}$ for each $\mathrm{kV}$ setting was determined using the automatic exposure control (AEC) of the X-ray unit. These exposure parameters were recorded and used as the standard while acquiring images for all three processing algorithms.

The SNRs were calculated for all of the steps using equation 1 . The value for $\mathrm{N}$ and $\sigma$ were obtained from a region-of-interest (ROI) of 100x40 pixels on each step. The ROI covered $50 \%$ of the area of a step, and was drawn in appropriate areas of approximately uniform signal strength in which artefacts were not noted.

The CNRs were calculated for all contrast steps, in the same ROIs as for the SNR calculation, from equation 2. The value of $\sigma$ in this case was obtained from the thicker step, which is the step with lowest signal strength (B in equation 2).

Object visibility with decreasing size and decreasing contrast was evaluated with visual assessment of images of a Perspex contrastdetail phantom placed on a $2.5 \mathrm{~cm}$ thick Perspex sheet. The phantom was made in-house by drilling a square array of small holes into the surface of a Perspex block. The holes varied in depth (creating variable low contrast spots) in the one direction, and in diameter in the other. Each contrast row had a centre and randomly positioned off-centre hole of each size. The contrast steps are produced by varying hole depths from 0.70 to $2.5 \mathrm{~mm}( \pm 0.05 \mathrm{~mm})$, and the hole diameters ranged from 0.40 to $2.30 \mathrm{~mm}( \pm 0.05 \mathrm{~mm})$ (verified with a micro meter gauge).

The phantom was imaged at $40 \mathrm{kV}$ using the small focal spot size. The images were scored on the 3 megapixel screen used for clinical evaluation. The number of centre circles of which the off-centre circle was also visible in each contrast row (i.e. the smallest object still visible for a certain contrast) was determined by three different observers. Observers were allowed to adjust the window level, window width and zoom settings for best object visibility. The average score from the three observers were used to calculate IQFs for each processing algorithm using equation 3.

Resolution was assessed using MTFs, calculated from images acquired at $40 \mathrm{kV}$ using the small focal spot size. An APG phantom was used ${ }^{8}$ that had small metal plates, rotated $1^{\circ}$, specifically for this purpose (Fig. 1). Several profiles were drawn across the edge of an MTF plate, each 1 pixel wide. The number of profiles needed for a 1 pixel shift in the edge owing to the angulation was determined from pixel values.

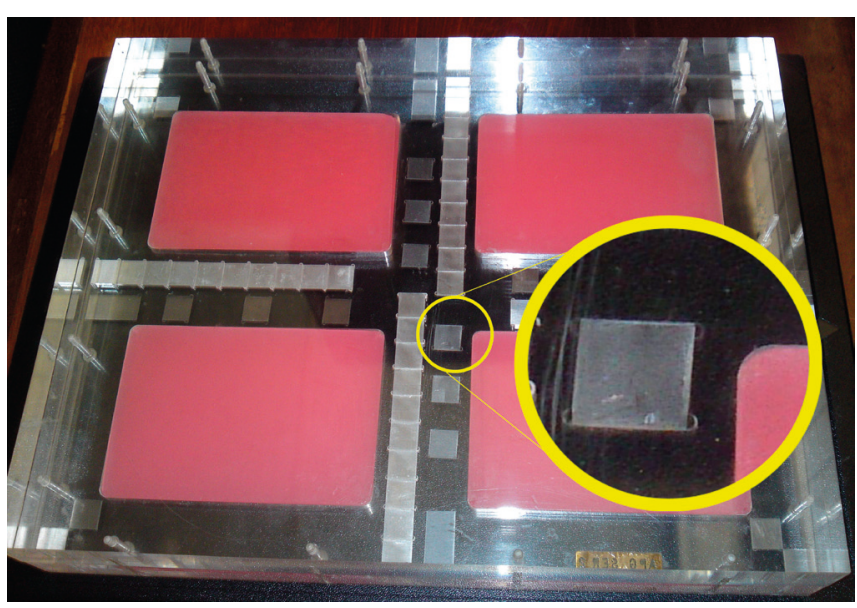

Fig. 1. The APG phantom used for MTF evaluation. The enlarged area shows an MTF plate.

A method to generate additional data points across the plate edge was used by the designers of the APG phantom, ${ }^{9}$ and is explained in the exaggerated illustration in Fig. 4. Each profile drawn provides a point on the edge with a slightly different pixel value. The displacement needed to align the pixel values with the real edge location was calculated using basic trigonometry, and the data sets for each profile were then shifted by this displacement. The equation is as follows:$$
x=\arctan (\alpha) \times i
$$

where $\alpha$ is the tilt angle of the plates $\left(1^{\circ}\right)$. This provides multiple data points across the edge profile.

The resulting curve is called the edge spread function (ESF), which is then smoothed and differentiated to obtain the line spread function (LSF), and the Fourier transform of the LSF was taken to obtain the MTF. 


\section{Results}

The SNRs on every step for all three processing methods at all energies were calculated, and are fairly similar. The results for energy of $70 \mathrm{kV}$ are shown in Fig. 2.

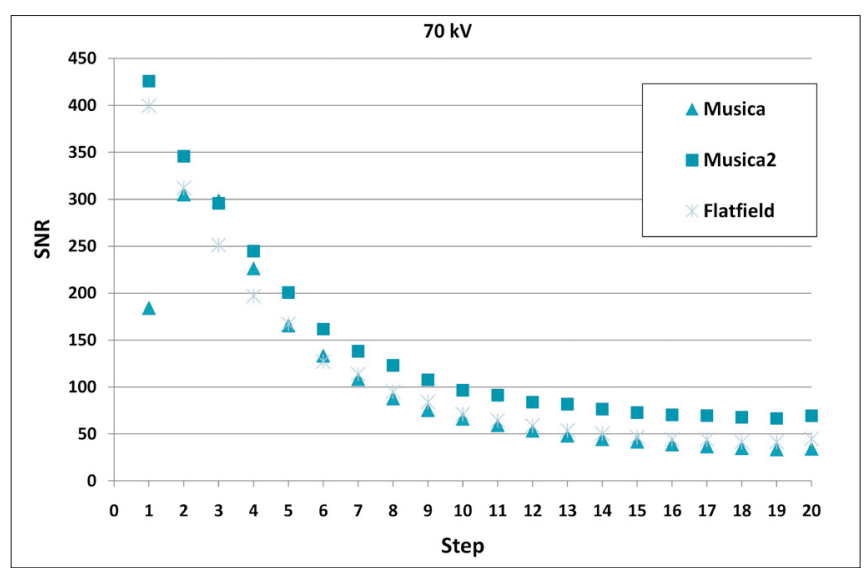

Fig. 2. Signal-to-noise ratios for all steps imaged at $70 \mathrm{kV}$. Readout artefacts can cause outliers as seen at step 1 (MUSICA).

The average CNRs between steps 1 and 4 were calculated and are shown in Fig. 3. Average CNRs were used to reduce errors introduced by artefacts (dust, CR defects). The specific steps used are the only steps clearly separable at all $\mathrm{kVs}$.

The contrast-detail images were scored by the three observers, and the results show that both MUSICAs allow better visualisation of small objects for all contrast conditions. The scoring of the observers was very similar. To quantify overall contrast-detail capabilities, a hyperbolic fit of the form as shown in equation 5 was used. A fit parameter $\left(h, x^{\prime}, y^{\prime}\right)$ fit was done on the data using the Solver Add-In in Excel:

$$
y+y^{\prime}=\frac{h}{x+x^{\prime}}
$$

The fitted curves are shown in Fig. 4. The IQFs calculated for the three processing algorithms are given in Table 1.

\begin{tabular}{|c|c|}
\hline & IQF \\
\hline Flatfield & 30.25 \\
\hline MUSICA & 24.75 \\
\hline MUSICA $^{2}$ & 23.75 \\
\hline
\end{tabular}

Profiles were drawn across the edge of the centre bottom MTF plate, and the positional displacements calculated using equation 4 . A smoothing function in Matlab (curve fitting tool; Loess, span=0.2) was applied to obtain the ESF, which was differentiated to get the LSF and the Fourier transform applied to calculate the MTF. The MTFs were calculated for the same MTF plate in all three images in the $\mathrm{x}$ - and $\mathrm{y}$-axes. The $\mathrm{x}$-axis is perpendicular to the cathode-anode direction of the X-ray tube. The MTFs calculated for the three algorithms are shown in Fig. 5.

\section{Discussion}

A number of observations were made from the SNR graphs. Firstly, the flattening off of the curve over the thick part of the wedge (step 20) is where the photons cannot penetrate the wedge, and therefore this effect diminishes at higher kV. Secondly, some outliers are seen (e.g. Fig. 2, step $1 \mathrm{MI}$ ), which are probably caused by artefacts (scratches on CR plate) in the image causing the average signal to be lower than it really is, and the standard deviation to be higher. Consequently, the ratio is reduced. The overall assessment over all energies shows MUSICA ${ }^{2}$ to be somewhat better than the other two methods regarding the SNR.

The CNRs are lower for the processed images than the flatfield, mainly owing to increased noise during the processing. There is also a downward trend as the energy increases, which is thought to be mainly due to the decrease in contrast caused by increasing the $\mathrm{kV}$, as photons become more penetrating. The noise also increases slightly, but the contrast has a bigger influence. For the higher energies, the CNRs are fairly constant, and the unprocessed images again show a higher CNR than the processed images, possibly owing to the noise increase in the processed images. MUSICA ${ }^{2}$ shows slightly higher CNR at lower $\mathrm{kV}$, while at higher $\mathrm{kV}$ no significant difference is seen between the methods.

The benefit of post-processing in visualising small objects over all contrast levels can be seen clearly from the contrast-detail graph (Fig. 4). The IQFs as shown in Table 1 indicate that MUSICA ${ }^{2}$ has slightly better image quality. Both processing methods show an improved ability to see low-contrast objects compared with the

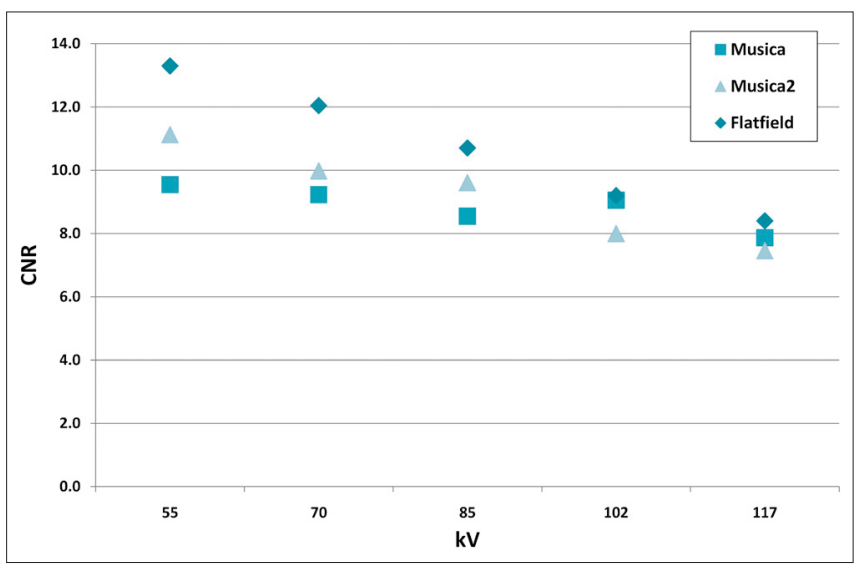

Fig. 3. Average contrast-to-noise ratios calculated from steps 1 to 4 of the step wedge at all energies.

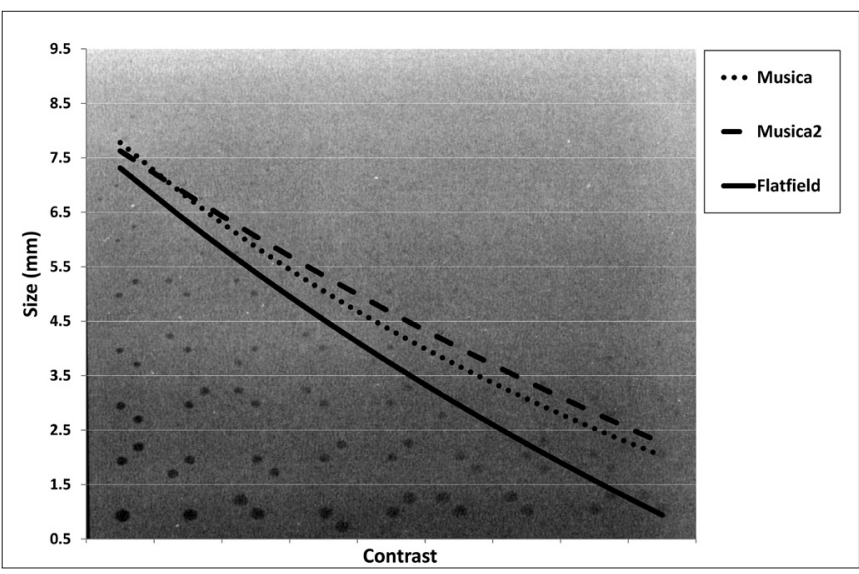

Fig. 4. Graph showing contrast with decreasing object size for the different algorithms. 


\section{ORIGINAL ARTICLE}

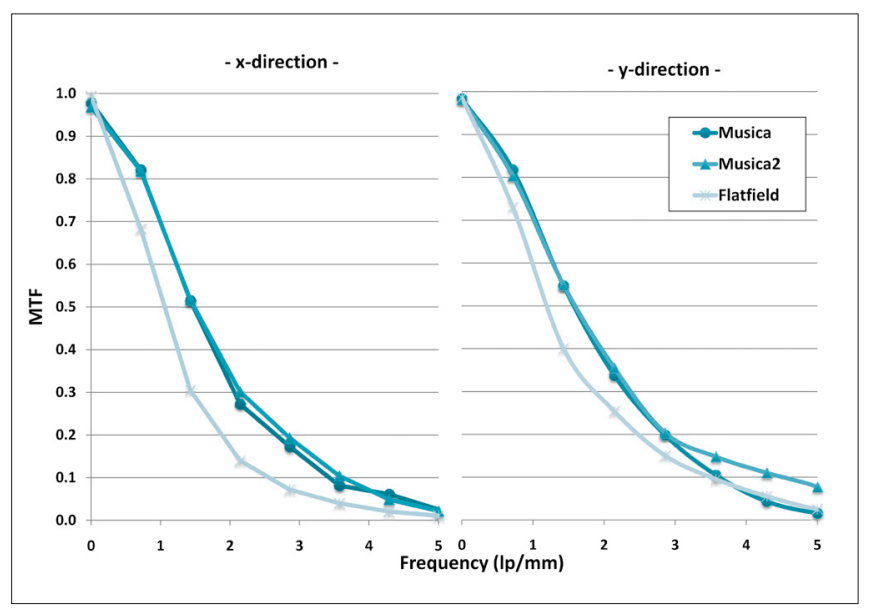

Fig. 5. Modulation transfer functions in both $x$ - and $y$-axes as calculated on the same MTF plate for all three algorithms. The post-processed images have higher MTF values over all useful frequencies.

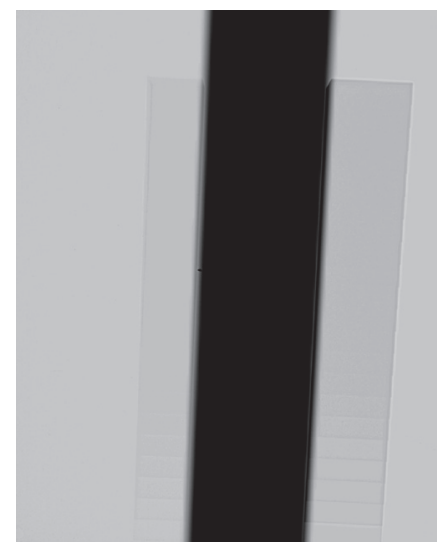

Fig. 6. Image of artefact encountered due to post-processing.
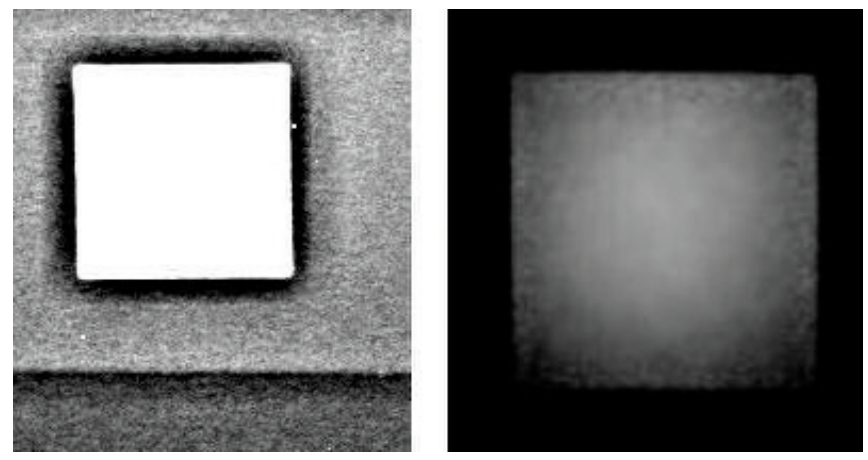

Fig. 7. The result of edge enhancement during software processing.

unprocessed image. Quantitatively this translates to objects of a size $10 \%$ smaller than with flatfield imaging being visible at all contrast levels using either of the MUSICAs. This is not an improvement in the spatial resolution capabilities of the system, but simply better visualisation of small objects owing to manipulation of the pixel values to enhance the contrast between the edges of small objects and their surrounding pixels. In other words, the transfer of frequencies is changed, as shown in the MTFs section. This is effectively selective signal amplification.

Both processing software packages show an increased transfer at almost all frequencies, the images therefore appearing visually sharper and overall resolution is improved (at sufficient contrast). The ability to see small objects better can be expected since the transfer of higher frequency information is better than for the flatfield image. The deviation from the trend in the y-axis of MUSICA ${ }^{2}$ at high frequencies is thought to be due to some high-frequency noise still present in the LSF, which gives a false impression of having higher MTF values.

The image shown in Fig. 6 shows a problem faced in automated software. This uniform black artefact area was created by the postprocessing software after encountering a perfectly straight edge throughout the length of the image.

An artefact commonly seen with post-processing is edge enhancement. This can be seen clearly in Fig. 7 (over-enhanced by window level/width adjustment), the square on the right illustrating how the area away from any edge is smoothed out while the edge is sharpened and so becomes noisier.

\section{Conclusion}

Both MUSICA software packages produced images with better contrast resolution than unprocessed images. The quantitative measurements indicate that MUSICA ${ }^{2}$ delivers marginally improved image quality over MUSICA. Both MUSICA packages show improved spatial resolution, which is the result of better visualisation of small objects owing to software manipulation of the pixel values. The MTF calculation might also include some noise, which does not represent real increased transfer of frequencies, and illustrates one of the complications faced in calculating MTFs. MUSICA ${ }^{2}$ has also shown slightly improved SNRs and CNRs at lower $\mathrm{kV}$; however, the images still appear more noisy.

It is also important to keep in mind that this study aimed at quantitative analysis of image quality differences between the software packages to provide an unambiguous comparison. To evaluate the system clinically, further investigation is necessary into all factors, including clinical image assessment by radiology experts, practical application aspects for the radiographers, cost, etc.

1. Bushberg J, Seibert J, Leidholt E, Boone J. The Essential Physics of Medical Imaging, 2nd ed. Philadelphia: Lippincott Williams \& Wilkins, 2002;255-287.

2. International Atomic Energy Agency (IAEA). Radiation Protection in Diagnostic and Interventional Radiology. http://www.iaea.org (accessed 3 December 2010).

3. Vuylsteke P, Schoeters E. Multiscale image contrast amplification (MUSICA). Proc SPIE 1994;167:551560

4. Schaetzing R. Agfa’s MUSICA2, Taking Image Processing to the Next Level. Mortsel, Belgium: Agfa Healthcare, 2007.

5. Vuylsteke P, Schoeters E. Image Processing in Computed Radiography. 1999. Paper 16, DGZfP proceedings, BB 67-CD, Germany.

6. Aichinger H, Dierker J, Joite-Barfuss S, Sabel M. Radiation Exposure and Image Quality in X-ray Diagnostic Radiology. Heidelberg, Germany: Springer Verlag, 2004:78-79.

7. Matlab - The Language of Technical Computing. V7. Natick MA, USA: Mathworks, 2004.

8. Hamza A, Alport MJ, Rae WID. The design and fabrication of a full field quantitative mammographic phantom. Journal of Science and Technology 2009;10(3):141-158.

9. Hamza A, Alport MJ, Rae WID. Qualitative and quantitative evaluation of the APG phantom. Journal of Science and Technology 2009;10(3):168-187. 